\title{
Prevention of the Spread of Viral Disease Using Artificial Intelligence from Data Obtained by UAVs
}

\author{
Katerina Prihodova ${ }^{1, *}$, and Jakub Jech ${ }^{1}$ \\ ${ }^{1}$ University of Pardubice, Faculty of Economics and Administration, Institute of System Engineering \\ and Informatics, Studentská 84, 53210 Pardubice, Czech Republic
}

\begin{abstract}
.
Research background: One of the significant globalization consequences is a threat of rapidly spreading communicable diseases. In recent months, COVID-19 has spread worldwide. It is a highly infectious disease, which is manifested mainly by fever, respiratory problems, muscle pain and fatigue. Therefore, there is a need to reliable monitor people's body temperature. If the monitoring process takes places in enclosed spaces, the procedure may be performed at the entrance to the building. However, the problem occurs in public spaces. Therefore, to solve this problem, we propose the use of a drone with a thermal camera for scanning people in public spaces and subsequent evaluation using classification methods.

Purpose of the article: The aim of this article is to create a model for sensing and measuring the body temperature of people in public spaces so that the global impacts of COVID-19 on the economy and society are reduced.

Methods: To monitor large areas, it is necessary to have suitable methods for obtaining quality data. One of the methods for obtaining data with the high spatial resolution is the use of UAVs with a planned flight. Artificial intelligence methods will be used for the classification of persons; their representatives are, e.g. convolutional neural networks.

Findings \& Value added: The proposed model of sensing and subsequent classification of people into groups (normal body temperature, elevated body temperature). The output of the model will help to monitor the spread of infectious diseases (the condition is a symptom - increased body temperature) in today's globalized world.
\end{abstract}

Keywords: consequences of globalization; $C O V-19$; classification; UAV; recognition

JEL Classification: $118 ; C 88 ; Q 56$

\footnotetext{
* Corresponding author: katerina.prihodova@student.upce.cz
} 


\section{Introduction}

One group of today's global risks are Societal risks (economic disparities, demographic challenges, migration, water and food security, infectious diseases) [1]. Nowadays, infectious diseases are a significant problem. Infectious diseases are caused by pathogenic microorganisms, such as bacteria, viruses, parasites or fungi. These diseases can be spread, directly or indirectly, from one person to another. Exist infectious diseases of animals that can cause disease when transmitted to humans called zoonotic diseases.

Today's globalized world brings excellent benefits in the fight against and information about diseases. Development and globalization also have disadvantages in the form of mutations in existing diseases and the emergence of new types of viruses/diseases and rapid spread.

COVID-19 is an infectious disease caused by the new coronavirus SARS-CoV-2 [2], which began spreading in December 2019 from the Chinese city of Wuhan [3]. COVID-19 has already spread all over the world, and there is no known functional cure for it yet. COVID-19 is a highly infectious disease. The virus spreads from person to person through droplets during sneezing, coughing, or body contact. The World Health Organization has estimated that the value of the R0 infectivity index can vary somewhere between 1.4-2.5, which is like SARS. [4] The incubation period is approximately between 1 and 14 days, and even during this time, the disease is infectious. Transmission of the virus to other persons was confirmed in the period 1-3 days before the onset of symptoms of the disease. The median incubation period is approximately 5 days. The disease has symptoms and course very similar to more severe influenza or other similar influenza diseases. It is therefore complicated to distinguish coronavirus infection from influenza at first sight. Therefore, it is currently the only possible way to detect the virus when undergoing a PCR test [5]. In PCR assays, nasopharyngeal and throat mucus samples are subsequently screened for RNA identical to SARS-CoV-2 coronavirus RNA.

The signs and symptoms are like other infectious diseases. The first is fever (in $83-99$ $\%$ of cases), great fatigue and shortness of breath. Later, a dry irritating cough (in $59-82 \%$ of cases) or muscle and joint pain is added. The vital signs of admitted patients are usually stable. Loss of smell (hence the taste) called anosmia (in 15 to $30 \%$ of cases) is also possible without other symptoms $[4,6]$. More severe cases can lead to pneumonia, acute inflammation of the heart muscle, organ failure and death. Children and young patients with the mild or asymptomatic disease often develop frostbite-like skin patches, rarely blisters, most commonly on the toes. These are a hidden symptom of an infection that is mild but indicates a possible virus carrier.

According to studies from March 2020, in $81 \%$ of cases, the disease is mild and does not require hospitalization (home treatment), in 14\% it can develop pneumonia, and $5 \%$ of those infected have a critical course with organ failure [2]. As with seasonal influenza, COVID-19 is asymptomatic in many infected patients.

The first symptom is an excellent point to create systems to prevent spreading of diseases or identify sick people. Elevated temperature is manifested throughout the patient's body and can be easily detected. A thermometer is used for contact measurement to determine the increased temperature, and a thermal camera can be used for non-contact measurement [7]. Non-contact measurement is better from a safety point of view because there is no direct contact between people.

Temperature measurement using a thermal imager is based on measuring the infrared part of the spectrum, where objects emit heat in infrared wavelengths. This measurement can be performed remotely. 


\section{Methodology}

The aim of this paper is to identify people with elevated body temperature in outdoor areas, where it is very difficult or impossible to guard all access roads to the place. By recognizing people with fever, it is possible to help solve a global problem such as infectious diseases. A model will be designed that uses UAV flight control with a thermal camera, and deep learning methods are used for subsequent image processing and classification.

As mentioned in the introduction, the symptom of elevated temperature can be measured non-contact using a thermal imager. The thermal imager can be easily placed at the entrance points of buildings. For monitoring the temperature of people in public spaces using thermal cameras, it is financially demanding at the entry points (many entry points). Therefore it is suitable to use a drone as a carrier for the thermal camera [8].

The drone quickly captured the entire territory. For high-quality drone scanning of public spaces, it is important to use a planned flight that guarantees full coverage of the area [9]. The planned flight will also be less time consuming compared to a comparable manual drone flight. There are specific rules for the planned flight, which relate to the longitudinal and transverse overlaps of the images and the flight level. Flying drones is subject to local legislation, smaller drones can fly over people, but for this kind of study, flying over people can be created as an exception. The ideal drone that meets the parameters for obtaining thermal images of people in public spaces is the DJI Mavic 2 Dual Enterprise drone.

The DJI Mavic 2 Dual Enterprise [10] is a small drone with 899 g MTOW (maximum takeoff weight) that contains two types of sensors in the camera. The first sensor is of the RGB type and the second is of the thermal type, the drone takes two images simultaneously, one of the images is in the visible spectrum, and the other is in the infrared spectrum.

For the flight, planning is used software developed by DJI called DJI Ground Station Pro (DJI GS Pro). DJI GS Pro is an iOS (iPad) freeware application and provides interactive flight planning and controlling flight itself [10]. To planning flight, its need, choose area and parameters of overlaps and flight altitude. The app itself set waypoints where is taken image and safe to the internal storage of a drone.

Method for drone flight planning [11] is more efficiency, cheaper and faster than a method of taking thermal images in entering points, these enter point of the public area must cover all area, and this cannot be completely guaranteed. Even though the drone flight planning method is fast, it is not fast enough to scan the whole area, and there is a redundancy of data (persons moving in the area). However, it does not matter for the final classification of infected persons, because the drone takes pictures in two spectra and then the infected persons from the thermal image can be compared with the persons from the image in the visible spectrum.

It is necessary to detect the faces of individual persons in the images obtained during the controlled flight. The face was chosen for the subsequent classification of persons into two categories (increased body temperature, normal body temperature) for purely practical reasons. Face recognition is very far in practical applications. Facial analysis has been used in the last two decades for the identification of persons, gender recognition, ethnic origin, age estimation $[12,13]$. At the same time, it is a part of the body for which it is relatively easy to get a picture of it, and there is no need for the cooperation of recognized persons.

The model uses a Faster R-CNN detector for face detection. It is a single, unified network for object detection. It is one of the most modern networks for object detection in an image using regional proposal algorithms. Faster R-CNN is shown in Figure 1. [14] 


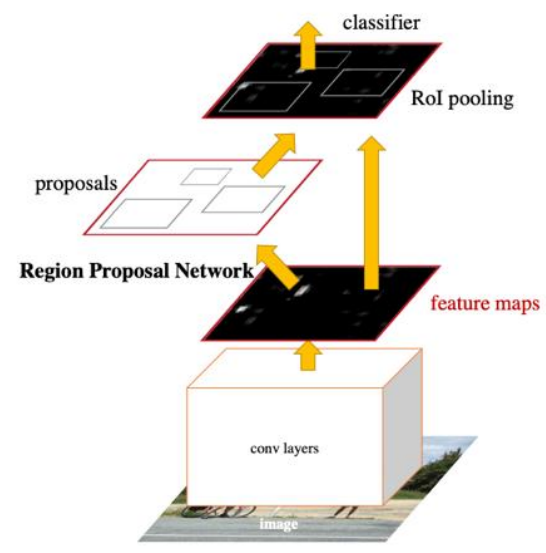

Fig. 1. Faster R-CNN detector for face detection. Source: [14]

After detecting a face, it is necessary to classify the detected face. The convolutional neural network GoogLeNet is used in the classification model. Convolutional neural networks first extract functions and then classify. All CNNs have a typical basic architecture, shown in Figure 2. The first layer is the input layer; the input is an image of size $\mathrm{m} \mathrm{x} \mathrm{m} \mathrm{x} \mathrm{n.}$ The input size for GoogLeNet is $224 \times 224 \times 3$. Hidden layers follow. The convolution layer together with the activation function is used to extract functions, the deeper the convolution layer in the network, the more complex the function is extracted. Convolution layers are followed by pooling layers, which are used to reduce the image. The extraction of functions is followed by classification using the fully connected layer - Softmax. GoogLeNet is composed of 22 layers.

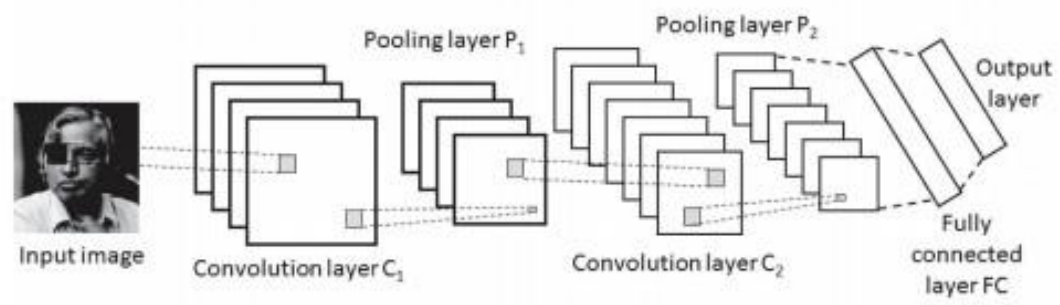

Fig. 2. Typical Convolutional Neural Network architecture. Source: [15]

\section{Results}

In the proposed model of recognizing people with a symptom of infectious diseases using UAVs, the flight planning of UAVs with a thermal camera is first used. Thermal images of persons are obtained, from which faces are detected using a Faster R-CNN detector. The thermal image of the face is processed, followed by feature extraction. This is followed by classification into two groups according to the body temperature of the face. If a person has a fever is classified as a person with a symptom of an infectious disease. If he does not have a fever, he will be classified as a person without a symptom of an infectious disease. This principle is illustrated by the flowchart in Figure 3. 


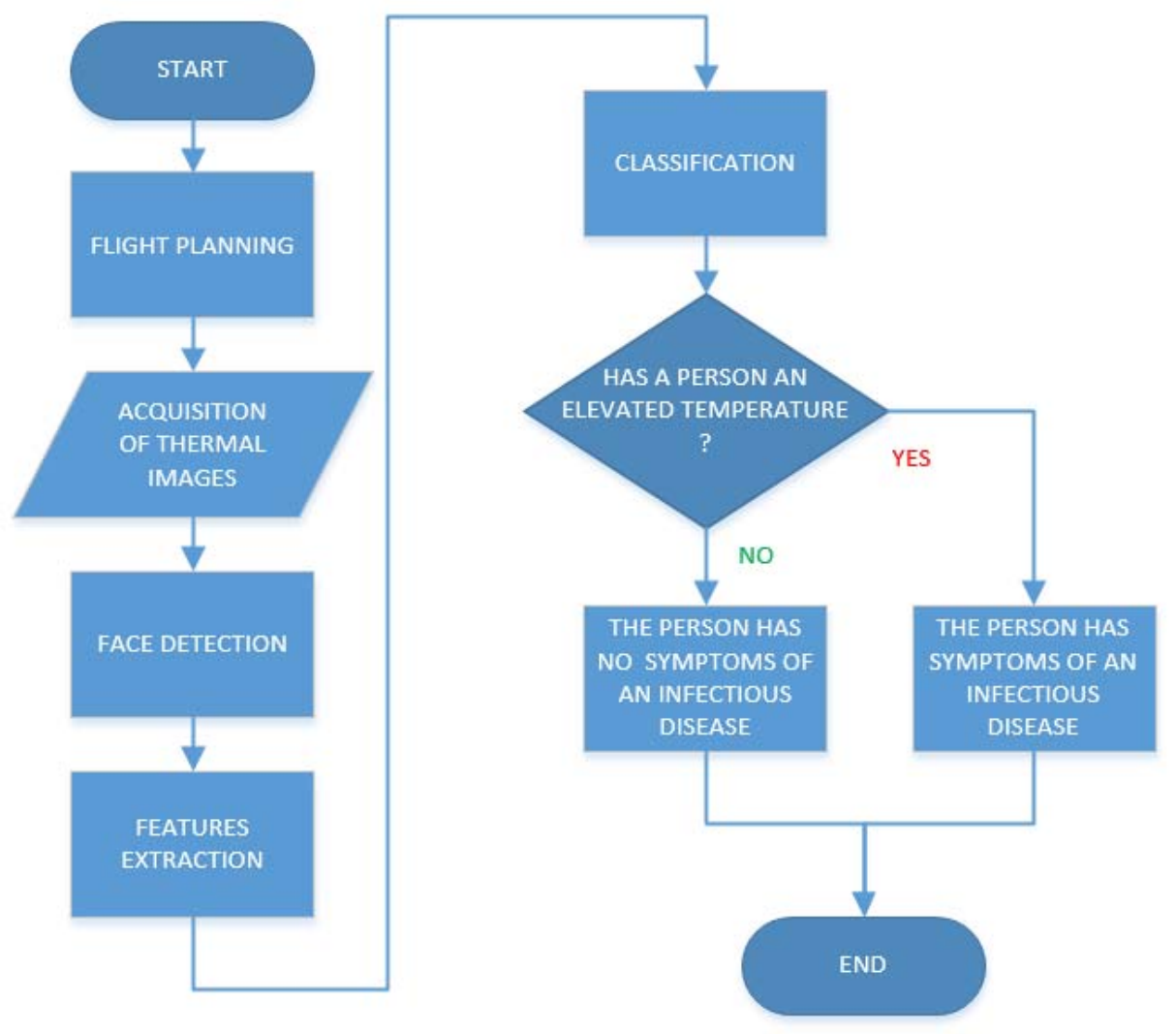

Fig. 3. Flow chart of the proposed model. Source: Authors.

\section{Discussion}

As already mentioned, the use of UAVs with a thermal camera to monitor people in public places to identify people who have a fever, which is a symptom of many infectious diseases, has vast potential in real applications.

In this article, a model was proposed in which a UAVs with a thermal camera and flight planning are used to monitor people in public spaces where it is difficult or impossible to check people on entry. This article also considers the quality of images from available thermal imagers placed on a UAV carrier and the associated flight level to obtain quality images. Subsequently, the faces of individual persons are detected on the obtained thermal images using a faster R-CNN detector. In the next step, the detected faces are classified into two groups (increased body temperature, normal body temperature) using the convolutional neural network GoogLeNet.

Further research can be focused on contact retrieval; this can be done when a video is acquired during the planned UAVs flight. Retrospectively, they will be able to find out the contacts of people with elevated temperature.

With the ever-improving parameters of thermal cameras and lower price, it is also possible to focus on research in which a better thermal camera would be used. Better 
parameters of thermal cameras will enable shooting from a greater distance, therefore with the help of UAV and from a higher flight level, which brings a safer feeling for the subjects.

This article was supported by grant SGS_2020_17 and SGS_2020_018 supported by the Student Grant Competition.

\section{References}

1. Helbing, D. (2013). Globally networked risks and how to respond. Nature, 497(7447), 51-59.

2. Coronavirus Symptoms (COVID-19) (2020, April 24). Worldometer. Retrieved from: https://www.worldometers.info/coronavirus/coronavirus-symptoms/\#mild

3. Hadi, A. G., et al. (2020). A Review on COVID-19: Origin, Spread, Symptoms, Treatment, and Prevention. Biointerface Research in Applied Chemistry, 10(6), 72347242.

4. Infectious diseases (2020). World Health Organization. Retrieved from: https://www.who.int/topics/infectious_diseases/en/

5. Täufer, M. (2020). Rapid, large-scale, and effective detection of COVID-19 via nonadaptive testing. Journal of Theoretical Biology, 506, 110450.

6. Mei, X., Zhang, Y., Zhu, H., Ling, Y., Zou, Y., Zhang, Z., et al. (2020). Observations about symptomatic and asymptomatic infections of 494 patients with COVID-19 in shanghai, china. American Journal of Infection Control, 48(9), 1045-1050.

7. Alessio, K. O., Tischer, B., Voss, M., Teixeira, I. D., Brendler, B. M., Duarte, F. A., (2020). Open source, low-cost device for thermometric titration with non-contact temperature measurement. Talanta (Oxford), 216, 120975.

8. Rudol, P., \& Doherty, P. (2008). Human body detection and geolocalization for UAV search and rescue missions using color and thermal imagery. In: 2008 IEEE aerospace conference, 1-8.

9. Zhao, X., Luo, Y., \& He, J. (2020). Analysis of the thermal environment in pedestrian space using 3D thermal imaging. Energies (Basel), 13(14), 3674.

10. DJI. (2020). DJI. Retrieved from: https://www.dji.com/

11. Liao, S., Zhu, R., Wu, N., Shaikh, T. A., Sharaf, M., \& Mostafa, A. M. (2020). Path planning for moving target tracking by fixed-wing UAV. Defence Technology, 16(4), 811-824.

12. Carletti, V., Greco, A., Percannella, G., \& Vento, M. (2020). Age from faces in the deeplearning revolution. IEEE Transactions on Pattern Analysis and Machine Intelligence, 42(9), 2113-2132.

13. Prihodova, K. \& Jech, J. (2019). Low-cost system for gender recognition using convolutional neural network. In Proceedings of 34th International Business Information Management Association Conference. Vision 2025: Education Excellence and Management of Innovations through Sustainable Economic Competitive Advantage, IBIMA 2019. International Business Information Management Association-IBIMA, 6316-6322.

14. Ren, S., He, K., Girshick, R., \& Sun, J. (2017). Faster R-CNN: Towards real-time object detection with region proposal networks. IEEE Transactions on Pattern Analysis and Machine Intelligence, 39(6), 1137-1149.

15. Prihodova, K., \& Hub, M. (2020). Hand-Based Biometric System Using Convolutional Neural Networks. Acta Informatica Pragensia, 9(1), 48-57. 